\title{
On words and their letters
}

\author{
TATJANA A. NAZIR, J. KEVIN O'REGAN, and ARTHUR M. JACOBS \\ CNRS, Université René Descartes, Paris, France
}

\section{(C. W. Eriksen, Sponsor)}

\begin{abstract}
In a recent article, McConkie et al. (1989) proposed that, due to the rapid drop-off of visual acuity, the amount of visual information available from a word is maximal when the eye fixates the middle of the word, and decreases on both sides of this optimal viewing position with each letter of deviation. However, data on perceptual span have demonstrated that during a fixation, more letters are utilized to the right than to the left of the fixation point, which would predict that the optimal viewing position should be left of center. In a letter discrimination task, the ratio of the left/right asymmetry was determined and the probability of correct word recognition as a function of fixation location in the word was estimated. The predictions were highly compatible with empirical results on word recognition.
\end{abstract}

Recent studies in reading and word recognition have shown that eye-movement behavior depends strongly on the position in the word where the eye first fixates (McConkie, Kerr, Reddix, Zola, \& Jacobs, 1989; O'Regan, 1984, 1989; O’Regan \& Lévy-Schoen, 1987; O'Regan, Lévy-Schoen, Pynte, \& Brugaillère, 1984; Vitu, O’Regan, \& Mittau, 1990). The probability of refixating in a word is lowest when the eye fixates near the middle of the word, and increases to both sides of this "optimal landing" or "optimal viewing" position with each letter of deviation, resulting in a U-shaped "refixation curve" (McConkie et al., 1989). The primary basis for the optimal-landing-position phenomenon is seen to be the dramatic drop-off of visual acuity that is found even within the fovea. Since minimum angle of resolution increases as a linear function of the distance from the center of vision (Jacobs, 1979; Olzak \& Thomas, 1986), in a recent article McConkie et al. (1989) proposed a simple summed-letter information model of word identification which can account for the obtained empirical results. The model has the following assumptions: (1) The frequency of identifying a word during initial fixation is a linear function of the amount of visual information available from it. (2) The total visual information obtained from a word is the sum of the information available from all its letters. (3) The amount of visual information obtained from a letter decreases as a linear function of its distance from the center of vision.

Given the second and the third assumptions, the amount of visual information that can be extracted from a word varies as a function of the position of the eye in the word: a maximum of visual information can be extracted when the eye is at the center of the word. However, when the

This work was supported by a grant from the Fondation FYSSEN to the first author. The authors' mailing address is Groupe Regard, Laboratoire de Psychologie Expérimentale, CNRS, 28 rue Serpente, 75006 Paris, France.

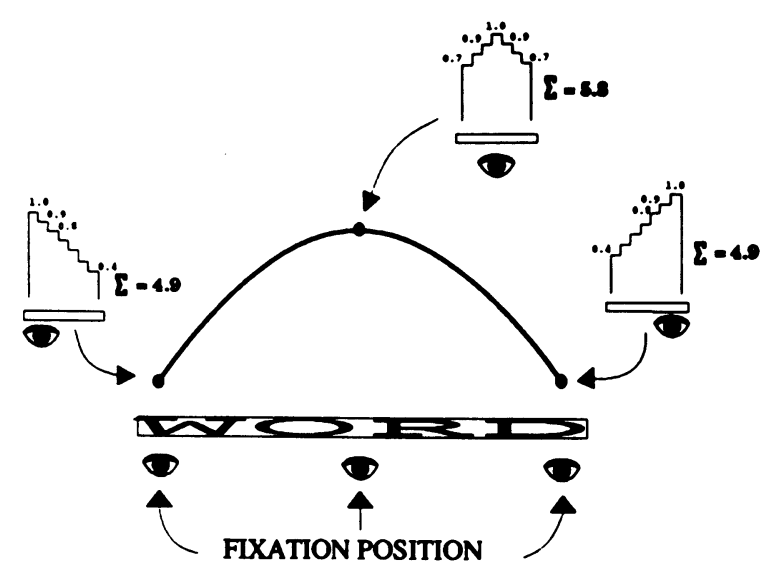

Figure 1. Distribution of available visual information as a function of fixation location in a word as suggested by McConkie et al. (1989). The amount of visual information available from the directly fixated letter is equal to 1 . This information drops by an arbitrarily chosen value of .1 for each letter position of eccentricity. The three histograms show the amount of information obtained from every letter of the word while the first, the middle, or last letter of the word are being fixated. By summing up the letter information (histogram) obtained at the different positions in the word, the curve gives the total amount of visual information obtained from a word while the different positions in the word are being fixated.

position of the eye is shifted to one side or the other, the total amount of available information decreases. The calculation suggested by McConkie et al. is illustrated in Figure 1 . The underlying assumption is that the amount of visual information available from a directly fixated letter is equal to 1 , and that with each letter position of eccentricity, this information drops by an arbitrary, chosen value of 0.1 . Because the available information is assumed to drop off equally quickly going leftwards or rightwards, the curve in Figure 1 showing the total available information is symmetric around the word center. 
However, classical results on perceptual span have demonstrated a left/right asymmetry of the number of letters utilized during a fixation. To the left of fixation, only a few letters are used; to the right, however, the perceptual span probably extends to 10-15 letters (McConkie \& Rayner, 1976; Rayner, Well, \& Pollatsek, 1980; Rayner, Well, Pollatsek, \& Bertera, 1982; Underwood \& McConkie, 1985). A number of other studies have shown that although the visibility of isolated letters is the same in the left and the right visual field (e.g., Bouma \& Legein, 1977), when letters are presented within strings of other letters, there is a superiority of the right visual field (e.g., Bouma, 1973; Bouma \& Legein, 1977). These facts suggest that McConkie et al.'s calculation should perhaps be modified to take into account this asymmetry. It is clear that if information drops off faster going leftwards than going rightwards, then we can expect the predicted refixation curves no longer to be symmetric, since it will be advantageous to fixate slightly left of center so that fewer letters suffer the fast drop-off rate.

In the following experiment, we determined the ratio of left/right asymmetry in discriminating letters at various eccentricities in a letter string. Using this ratio, and following the model proposed by McConkie et al. (1989), we estimated the optimal viewing position in words. We then compared this estimation with empirical data on word recognition.

\section{EXPERIMENT 1}

\section{The Ratio of the Left/Right Asymmetry}

To get an empirical estimate of the rate of information loss going leftwards as compared with that going rightwards, we chose a letter discrimination task in which the subject had to discriminate, from two possible distractors, a target letter presented at different locations in a nineletter string of $k \mathrm{~s}$. To be able to collect discrimination data for both sides of fixation, we measured performance while the eye was fixating either the first letter (thus, the target appeared to the right) or the last letter of the string (the target appeared to the left).

\section{Method}

Subjects. Twelve subjects with normal or corrected-to-normal vision participated in the experiment.

Material. Strings of nine lowercase letter $k$ s served as stimuli. In each of the strings, one letter was replaced by one of the following letters: $c, o, f$, or $t$. These critical letters could appear at any one of the possible nine letter positions. The letters were defined in an $8 \times 8$ pixel matrix that subtended $.3^{\circ}$ at a viewing distance of $50 \mathrm{~cm}$.

Design. The experiment contained two blocks. In each block, the subject's task was to discriminate one prespecified target letter from two possible distractors by pressing a response key. For half of the subjects, the targets were the letters $c$ and $t$ (one in each block) and the distractors were the remaining two letters $o$ and $f$ (in both blocks); for the other half, the target letters were $o$ and $f$ and the distractors were $c$ and $t$. The homogeneity of the string allowed the position of the critical letter to be detected easily, and the discrimination task forced the subject to identify the target rather than just report the presence of a letter different from the background-string letters. In each of the blocks, the target letter and each of the two distractors appeared 10 times at each possible location in the string to both sides of fixation location. Thus, there were
540 trials per block. The position of the critical letter in both the string and the presentation side of the string (right or left of fixation) was randomized.

Procedure. A fixation point appeared at the center of the screen for $500 \mathrm{msec}$ and then was replaced by a letter string, which appeared such that the directly fixated location was either the first or the last letter of the string. In order to collect enough omission errors, the presentation duration of the stimulus string was reduced to $20 \mathrm{msec}$ and followed by a mask consisting of a string of nine \#s.

\section{Results and Discussion}

Figure 2 gives the results of the letter-in-string discrimination experiment. As shown by the regression lines, the probability of detecting a letter drops off faster on the left than on the right by a factor of $.11 / .06=1.8$. When, as shown in Figure 3, this ratio is used in the McConkie

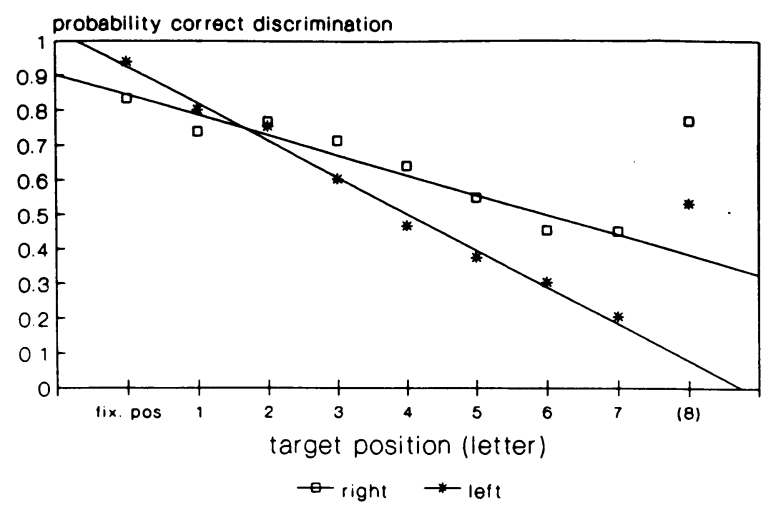

Figure 2. The rate of information loss going leftwards (stars) as compared with that going rightwards (squares) as a function of target eccentricity. The regression line when the target had to be identified to the right of fixation $(Y=0.9-0.06 X ; r=-0.97)$ and when it had to be identified to the left of fixation $(Y=1.03-0.11 X$; $r=-0.99)$. Because contour interaction is different for the last letter position of the string, this position-(8) - was excluded from the calculations of the regression lines.

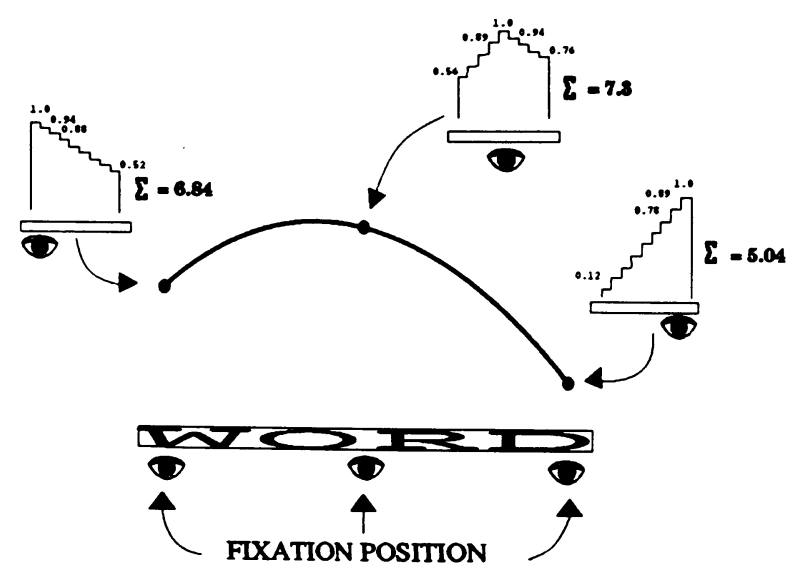

Figure 3. This figure is similar to Figure 1 except that the empirical values for the rate of information loss obtained in the letter discrimination experiment have been used to compute the curve. Visual information drops by a value of .06 for each letter position of eccentricity to the right and one of $\mathbf{. 1 1}$ for each letter position of eccentricity to the left of fixation. 
et al. calculation, it appears that the maximum amount of information should be available from a word when it is fixated slightly left of the center of the word.

Although the calculation proposed by McConkie et al. (1989) is very appealing, it has the disadvantage of using the notion of "information," which has not been properly defined in the present context. A more natural approach would perhaps be to use probabilities.

The data provided by the letter-in-string discrimination experiment suggest that the probability that a letter can be reported falls off by 0.06 per letter on the right and by 0.11 per letter on the left. Assuming that under normal reading conditions the probability of recognizing the directly fixated letter is 1 , these results might be assumed to suggest that the probability of recognizing the successive letters going rightwards would be $0.94,0.88,0.82$, and so on, and of those going leftwards, $0.89,0.78,0.67$, and so on. The probability of recognizing all the letters of a nine-letter word can be calculated by multiplying together all the probabilities from the individual letters. This calculation is illustrated in Figure 4.

A problem with this calculation is that the data used correspond to conditions of very rapid, masked presentation, which is not the natural situation in normal reading. The actual drop-off rate of 0.6 and 0.11 measured

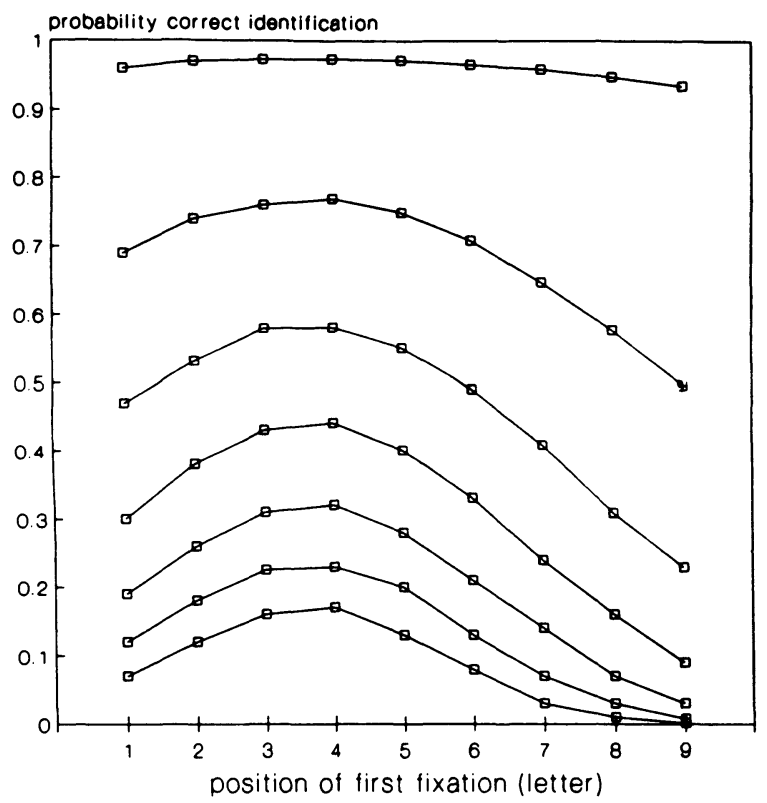

Figure 4. Predicted probability of correct identification as a function of fixation location in a word. The probability of recognizing the directly fixated letter is assumed to be 1 . The probability of recognizing the successive letters going rightwards drops by a value of .06 and going leftwards by a value of .11 for each letter position of eccentricity (bottom curve). The curves are obtained by multiplying the probability of correct letter identification at a given fixation position. The parameter distinguishing the different curves is the drop-off rate. The left-right ratio of $1.8 / 1$ is preserved in all curves. The lowest curve is calculated by using the empirically obtained dropoff rate of 0.11/0.06. For the other curves, the drop-off rate was successively reduced to $0.09 / 0.05,0.072 / 0.04,0.055 / 0.03,0.036 / 0.02$, $0.018 / 0.01$, and $0.0018 / 0.001$. here should therefore probably not be directly transposed to normal word recognition, where viewing conditions are much better and the drop-off will be slower. However, it seems reasonable to suppose that the ratio of drop-off going rightwards as compared with that going leftwards might be an invariant of the visual system and not dependent on the particular stimulus conditions. Figure 4 therefore shows curves obtained by using different values of the drop-off rates, while preserving the left-right ratio of 1.8/1. The absolute value of the drop-off rate slightly alters the shape of the curve: the smaller the drop-off rate, the flatter the curves become. However, the location where the curve has its maximum is not changed, and always lies one letter left of middle. (The drop-off rate of 0.11 on the left and 0.6 on the right obtained in the stringof- $k \mathrm{~s}$ experiment is the lowest curve in the figure.)

An interesting aspect of the present calculation is that it considers only visual factors. However, lexical constraints are undoubtedly active in word recognition, and these will substantially improve recognition probability. But, unless there are differences in the informativeness of different word parts, the effect of lexical constraints will be only to raise and lower the vertical position of the curves in Figure 4, and will not alter the position of the maximum. The difference between the calculated curve and a curve obtained for real word recognition (under comparable visual conditions) might be used as an estimation of lexical constraints.

What evidence is there to suggest that word recognition probability depends on the predicted asymmetric way on fixation location? Data from the literature has generally concerned gaze duration (O'Regan \& Lévy-Schoen, 1987; O'Regan et al., 1984; Vitu et al., 1990) or naming latencies (O'Regan et al., 1984) in tasks where the eye is free to move after the word appears. These data are compatible with the present predictions in showing an asymmetry, with improved word naming or gaze duration performance when the eye starts from the left of the middle of words. However, more directly compatible with the present predictions would be a measurement of word recognition probability rather than oculomotor performance. Therefore, in a second experiment, we measured probability correct lexical decision as a function of fixation location in a word.

\section{EXPERIMENT 2 Probability Correct Lexical Decision}

\section{Method}

Subjects. Twenty-five subjects with normal or corrected-to-normal vision participated in the experiment.

Material. Fifty nine-letter high-frequency words (median $=2,156$ per million; lower limit $=1,058$; upper limit $=7,931$ ), chosen from the Tresor de la langue Française (1971), and 50 nonwords served as stimuli. The nonwords were created by randomly replacing one or two letters in nine-letter words (different from the test words).

Procedure. On each trial, the subjects fixated a letter-high gap between two vertically aligned fixation lines. After $500 \mathrm{msec}$, the lines were replaced by a stimulus string which could form either a word or a nonword. The stimulus string appeared to be shifted horizontally in such a way that upon its appearance, the subject's eye was positioned on one 


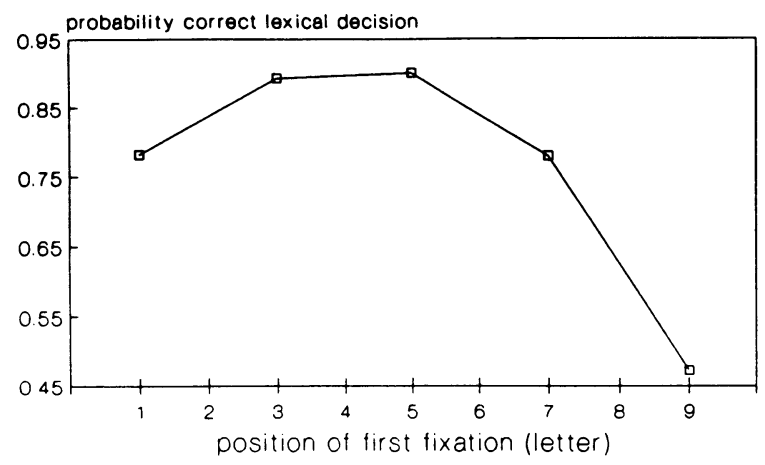

Figure 5. Probability of correct word recognition in the lexical decision task of Experiment 2 as a function of fixation position in the word.

of the odd-numbered letters of the string (see O'Regan et al., 1984). Each subject saw 10 words, presented at each of the five different fixation locations. Across all subjects, each word was seen an equal number of times from each fixation location. The subjects never saw the same word twice. To prevent subjects from making more than one fixation, presentation duration was limited to $170 \mathrm{msec}$. The subjects' task was to decide whether or not the stimulus was a word and to respond by pressing a response key. Probability correct recognition was measured.

\section{Results and Discussion}

In Figure 5, the probability of a correct lexical decision is given as a function of fixation location in the word. A clear effect of fixation position on performance is apparent, and the curve shows the expected asymmetry. A comparison with Figure 4 reveals a striking correspondence between the curves. The course of the curves is almost identical, with a maximum between the third and fifth letters and a decrease more toward the end than toward the beginning. The locations where we predicted a maximum of visual information or highest recognition probability were also the positions where the probability of correct lexical decision was highest.

\section{CONCLUSION}

It is interesting to note a difference between the present data and those obtained in normal reading by McConkie et al. (1989). Whereas we observe an asymmetry, with the optimal position being left of center, as predicted from our informational arguments, McConkie et al. found fairly symmetrical curves. The absence of asymmetry in refixation curves obtained by McConkie et al. may well be due to oculomotor factors that intervene while saccading from one word to another. In a recent study, Nazir (in press) showed that even when linguistic factors are set aside, the position of the eye in a letter string can itself affect eye-movement behavior. The probability of making a refixation (regardless of information extraction) is much higher in the first half of a letter string than in the second half. This tendency might have compensated for the asymmetry predicted from our informational or probability arguments and made McConkie et al.'s refixation curves symmetrical again.

The present results are suggestive as to the role of letters in word recognition. If the obtained "word-recognition curve" in Figure 5 really reflects available visual information, then this would be clear evidence for the contribution of each letter to word recognition.

\section{REFERENCES}

Bouma, H. (1973). Visual interference in the parafoveal recognition of initial and final letters of words. Vision Research, 13, 767-782.

Bouma, H., \& Legein, C. P. (1977). Foveal and parafoveal recognition of letters and words by dyslexics and by average readers. Neuropsychologia, 15, 69-80.

JACOBS, R. J. (1979). Visual resolution and contour interaction in the fovea and periphery. Vision Research, 19, 1187-1196.

McConkie, G. W., KerR, P. W., Reddix, M. D., Zola, D., \& JACOBS, A. M. (1989). Eye movement control during reading: II. Frequency of refixating a word. Perception \& Psychophysics, 46, 245-253.

MCConkIE, G. W., \& RAYNER, K. (1976). Asymmetry of the perceptual span in reading. Bulletin of the Psychonomic Society, 8, 365-368.

NAzIR, T. A. (in press). On the role of refixations in letter strings: The influence of oculomotor factors. Perception \& Psychophysics.

OlzaK, L. A., \& Thomas, J. P. (1986). Seeing spatial patterns. In K. R. Boff, L. Kaufman, \& J. P. Thomas (Eds.), Handbook of perception and human performance: Vol. 1. Sensory processes and perception (pp. 7.1-7.56). New York: Wiley.

O'Regan, J. K. (1984). How the eye scans isolated words. In A. G. Gale \& F. Johnson (Eds.), Theoretical and applied aspects of eye movement research (pp. 159-168). Amsterdam: Elsevier-North-Holland.

O'REGAN, J. K. (1989). Visual acuity, lexical structure, and eye movements in word recognition. In B. Elsendoorn \& H. Bouma (Eds.), Working models of human perception (pp. 261-292). London: Academic Press.

O'RegAN, J. K., \& LÉvy-SCHOEN, A. (1987). Eye movement strategy and tactics in word recognition and reading. In M. Coltheart (Ed.), The psychology of reading, attention, and performance XII (pp.363383). Hillsdale, NJ: Erlbaum.

O’Regan, J. K., \& Lévy-Schoen, A., Pynte, J., \& Brugaillère, B. (1984). Convenient fixation location within isolated words of different length and structure. Journal of Experimental Psychology: Human Perception \& Performance, 10, 250-257.

Rayner, K., Well, A. D., \& Pollatsek, A. (1980). Asymmetry of the effective visual field in reading. Perception \& Psychophysics, 27, 537-544.

Rayner, K., Well, A. D., Pollatsek, A., \& Bertera, J. H. (1982). The availability of useful information to the right of fixation in reading. Perception \& Psychophysics, 31, 537-550.

Tresor de la langue Française (1971). Nancy, France: Centre National de la Recherche Française.

UNDERWOOD, N. R., \& MCConkIE, G. W. (1985). Perceptual span for letter distinctions during reading. Reading Research Quarterly, 20, 153-162.

Vitu, F., O'Regan, J. K., \& MitTaU, M. (1990). Optimal landing position in reading isolated words and continuous text. Perception \& Psychophysics, 47, 583-600.

(Manuscript received October 24, 1990.) 